\title{
ANÁLISIS BIBLIOMÉTRICO DE LA REVISTA DUAZARY EN EL QUINQUENIO 2012-2016
}

\section{BIBLIOMETRIC ANALYSIS OF THE DUAZARY JOURNAL IN THE PERIOD 2012-2016}

\section{TÍTULO CORTO: ANÁLISIS BIBLIOMÉTRICO DE LA REVISTA DUAZARY}

\section{Oskarly Pérez-Anaya ${ }^{1}$, Guillermo Augusto Ceballos-Ospino², Diana Milena González-Gélvez ${ }^{3}$, Jesús David Suescún-Arregocés ${ }^{4}$}

Recibido en diciembre 06 de 2016

Aceptado en febrero 20 de 2017

Publicado en línea en mayo 15 de 2017

\section{Resumen}

El objetivo de este trabajo es analizar la producción científica de la revista Duazary entre los años 2012-2016, a través de un estudio bibliométrico de tipo descriptivo transversal. Para ello, se analizaron 98 artículos a partir de indicadores como: tipología del artículo, número de artículos por año, afiliación de autores, índice de colaboración, disciplina, citaciones e idioma de publicación. Los resultados evidencian que las contribuciones internacionales han aumentado desde el año 2014 y que predominan los artículos clasificados en las áreas temáticas de ciencias de la salud y medicina clínica (95\%). Igualmente, se encontró que más del 50\% del total de los manuscritos corresponden a artículos originales, escritos en su mayoría en idioma español. De esta manera, se considera que publicar un mayor número de artículos en inglés constituye una oportunidad de mejora para la revista.

Palabras clave: revista; bibliometría; ciencias de la salud.

\section{Abstract}

This study aimed at analyzing the scientific information of the Duazary Journal, between 2012 and 2016. To this end, a descriptive and cross-sectional bibliometric analysis was performed, including 98 published papers, based on the following indexes: paper typology, number of papers per year, institutional affiliation, collaboration index,

1. Biólogo, especialista en gestión ambiental. Asistente editorial. Revista Duazary. Universidad del Magdalena. Santa Marta, Colombia. Correo: oskperez123@gmail. com - http://orcid.org/0000-0002-0701-7847

2. Psicólogo. Universidad Católica de Colombia. Especialista en Gerencia en servicios de salud y en auditoria de servicios de salud. Universidad Jorge Tadeo Lozano, investigador asociado, miembro del grupo de investigación Psicología y Salud. Universidad del Magdalena. Santa Marta, Colombia. Correo: guillermoceballos@ gmail.com - http://orcid.org/0000-0002-1568-7058

3. Ingeniera Industrial, magíster en Ingeniería Industrial. Universidad Industrial de Santander. Directora de transferencia de conocimiento y propiedad intelectual. Universidad del Magdalena. Santa Marta, Colombia. Correo: dianamilenagonzalez@gmail.com

4. Candidato a magíster en Psicología Social Comunitaria. Universidad de Buenos Aires. Psicólogo de la Dirección Territorial Caribe de Parques Nacionales Naturales de Colombia. Tutor del Instituto de educación a distancia y formación para el trabajo IDEA. Universidad del Magdalena. Santa Marta, Colombia. Correo: ps.suescun@ gmail.com 
topic, citations, and language. The results show that international contributions have increased since 2014 and papers classified in the areas of health sciences and clinical medicine (95\%) predominate. Likewise, it was found that more than $50 \%$ of the total manuscripts correspond to original research papers, written mostly in Spanish. Therefore, increasing the number of papers published in English constitutes an opportunity for the journal improvement.

Keywords: journal; bibliometrics; health sciences.

\section{INTRODUCCIÓN}

$E^{1}$ l análisis bibliométrico devela el paso a paso de los procesos investigativos, al igual que permite reconocer el valor real que tiene la actividad científica en determinada disciplina ${ }^{1}$. Es por ello que posee múltiples características que están ligadas con la publicación que, en últimas, es el producto final de dicha actividad ${ }^{2}$. El artículo científico en la actualidad es el medio más utilizado para la publicación científica, que se define como la suma de componentes de un escrito que representa los hallazgos de una investigación ${ }^{3}$. En ese orden de ideas, la publicación termina convirtiéndose en el resultado final y palpable de cualquier evento de investigación. Desde esta hermenéutica, la bibliometría y sus indicadores adquieren validez como herramienta de la medición científica; por lo tanto, las citas y referencias bibliográficas conforman un aspecto importante de la comunicación científica, por lo que el análisis de estas, es una de las secciones más estudiada de la bibliometría, que se emplea para el estudio del consumo de información de una región, país, institución, revista o cualquier otro ente que desee conocer la trascendencia de sus escritos teóricos ${ }^{4}$.

En definición, la bibliometría es la disciplina que permite el estudio cuantitativo de la producción científica, a través del análisis de su propia naturaleza y del trasegar de una ciencia en determinado periodo $0^{5-8}$. Los indicadores que esta utiliza se basan en el supuesto de que los estudios más relevantes son usualmente los más citados, mientras que los menos importantes son ignorados ${ }^{9}$. Es así que algunos autores sostienen que los indicadores bibliométricos brindan orientación con respecto al valor científico de una revista, es decir, permiten saber cuáles son los artículos y autores más consultados y, por ende, tienen o son investigaciones de mayor relevancia ${ }^{10}$. Sin embargo, la anterior premisa no siempre es del todo cierta, debido a que si se publican textos controvertidos, también pueden ser citados con el propósito de debatirlos sin que ello sea reflejo de su calidad.

Las revistas de carácter científico desarrollan un importante papel en la divulgación de las comunicaciones científicas, debido a que conforman la vía más expedita para la validación de conocimientos originales y, a su vez, hacen posible la difusión dentro de las comunidades científicas, lo que las categoriza como el principal canal de comunicación en diversas áreas del saber ${ }^{11}$.

El análisis bibliométrico cobra mayor protagonismo cuando es utilizado para la elaboración de lineamientos y políticas. Es el caso de las instituciones de educación superior, que buscan medir la visibilidad de sus investigaciones científicas a nivel internacional y utilizan esta herramienta como elemento de medición y monitoreo de la efectividad de las acciones implementadas ${ }^{12}$.

En este contexto, se presenta este trabajo, el cual analiza la producción científica de la revista Duazary entre los años 2012-2016. La revista Duazary es una publicación semestral desde el 2004, año en el cual se creó bajo la adscripción de la Facultad de Ciencias de la Salud de la Universidad del Magdalena, y publica artículos relacionados con las siguientes áreas temáticas: salud pública, cirugía, especialidades clínicas, epidemiología, educación médica y demás ramas afines, que se enmarcan en investigaciones originales e inéditas. A la fecha, recibe contribuciones en español e inglés y su principal objetivo es que sus publicaciones permitan la comprensión del proceso salud-enfermedad en el ámbito local, nacional e internacional. Así mismo, se encuentra a texto completo en Doaj, Dialnet, Emerging Sources Citation Index, Index Copernicus, $\mathrm{CAB}$ Abstracts, entre otras plataformas, que en conjunto conforman más de 30 bases, índices, directorios, etc., y que han permitido incrementar notablemente su visibilidad en los últimos años. 
En este estudio se plantea el siguiente objetivo: analizar la producción científica de la revista Duazary en el quinquenio 2012-2016, a través de la identificación de la tipología de los artículos, número de artículos por año, afiliación de autores, instituciones de origen, índice de colaboración, disciplina, citaciones e idioma de publicación.

\section{MATERIALES Y MÉTODOS}

\section{Diseño del estudio}

Estudio bibliométrico de tipo descriptivo transversal, en el cual se empleó como material de estudio los artículos publicados entre los años 2012-2016 de la Revista Duazary. Se tuvo acceso a estos a través de la plataforma en línea de la revista que es Open Access. Allí se consideraron tipologías como: Investigación Científica y Tecnológica, Reporte de Casos Clínicos, Revisión, Columna del estudiante y Reflexión. De este estudio fueron excluidas secciones como editoriales y suplementos.

\section{Obtención de datos}

Los datos fueron obtenidos directamente de la plataforma oficial de la revista. Posteriormente, se revisó cada uno de los artículos, de los cuales se extrajo la siguiente información: institución de afiliación de cada autor, país de la afiliación institucional, títulos de los artículos, citaciones, número de autores por institución, número de autores por artículo, idioma, número de artículos por edición, número de artículos por tipología y la disciplina tratada en cada uno de ellos. Esta información fue organizada en tablas de Excel para su análisis.

Así mismo, para la sistematización por disciplina, se utilizó la Clasificación de áreas científicas según la Organización para la Cooperación y el Desarrollo Económicos (OCDE), que subdivide la rama de Ciencias Médicas y de la Salud en cinco áreas: Medicina Básica, Medicina Clínica, Ciencias de la Salud, Biotecnología en Salud y otras Ciencias Médicas; áreas que consecuentemente se dividen en múltiples ciencias ${ }^{13}$. Para ello, se requirió de la participación de un experto en el área, encargado de clasificar los artículos de acuerdo con las áreas de la OCDE.

\section{Análisis estadístico}

El análisis de los datos fue llevado a cabo a través de la utilización del programa Microsoft Excel 2013, en donde se realizaron tablas de doble entrada con el propósito de disminuir los errores que pudieran presentarse durante el procesamiento de los indicadores. Para cuantificar las citas obtenidas por la revista se requirió la utilización del programa informático Publish Or Perish, versión 5.26.2.

\section{RESULTADOS}

Entre los años 2012-2016 se publicaron, en la revista Duazary, 98 artículos, de los cuales 54 (55\%) corresponden a la tipología de Investigación Científica y Tecnológica, 18 (19\%) a Reporte de Casos Clínicos, 14 (14\%) a Revisión, siete $(7 \%)$ a Columna del Estudiante (sección suprimida a partir del 2014) y cinco (5\%) a Reflexión. La siguiente figura presenta estos resultados: (Figura 1).

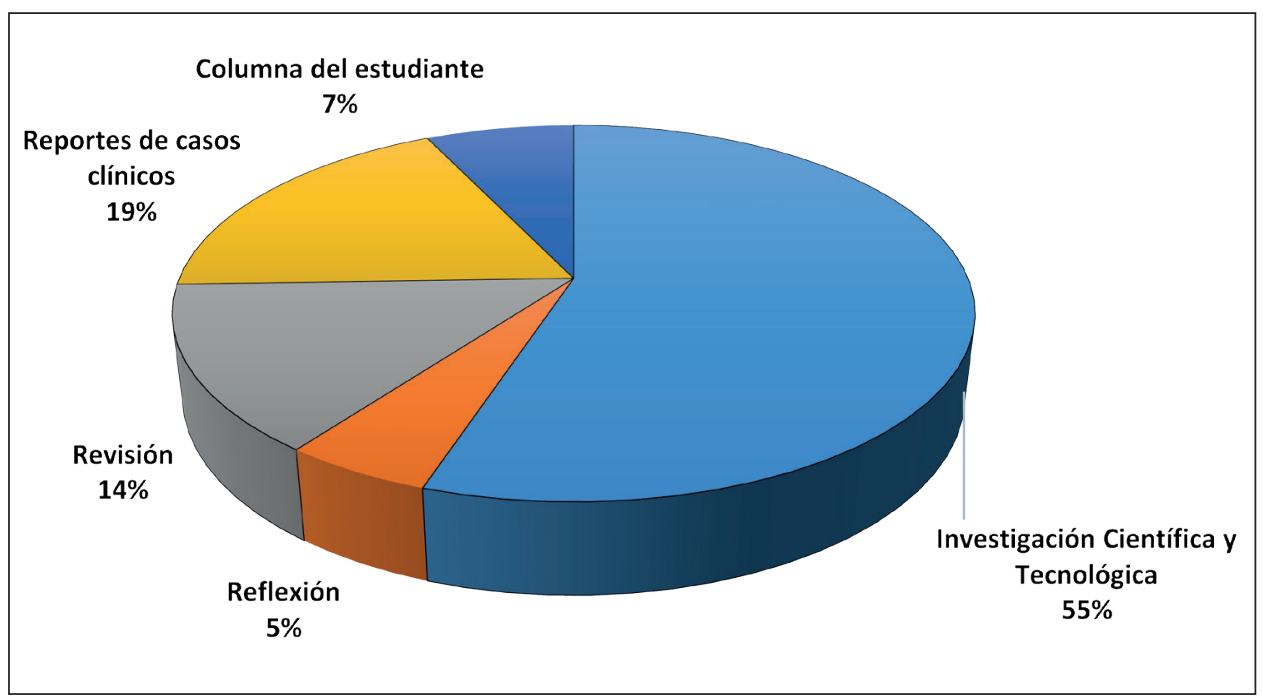

Figura 1. Tipología de los artículos publicados durante el quinquenio 2012-2016 en la revista Duazary. 
El número de artículos publicados por año fue variable, presentando un número mínimo de 15 y un número máximo de 27, en los años 2014 y 2012 respectivamente. En promedio se publicaron 19 artículos por año. En relación con el origen de las contribuciones, se encontró que en 33 de los artículos su primer autor contaba con afiliación interna o local, es decir, que provienen de la misma institución donde se edita la revista. También se encontró que 47 de las publicaciones son de origen nacional, proviniendo de hospitales, instituciones, fundaciones, etc., y que 17 artículos registran afiliación extranjera o internacional. De esta última aseveración, vale la pena destacar que se acrecentaron este tipo de escritos a partir de la edición 2014-2, manteniéndose constante en los números siguientes, e incluso llegando a desplazar la contribución de origen local. La siguiente figura presenta los anteriores resultados: (Figura 2).

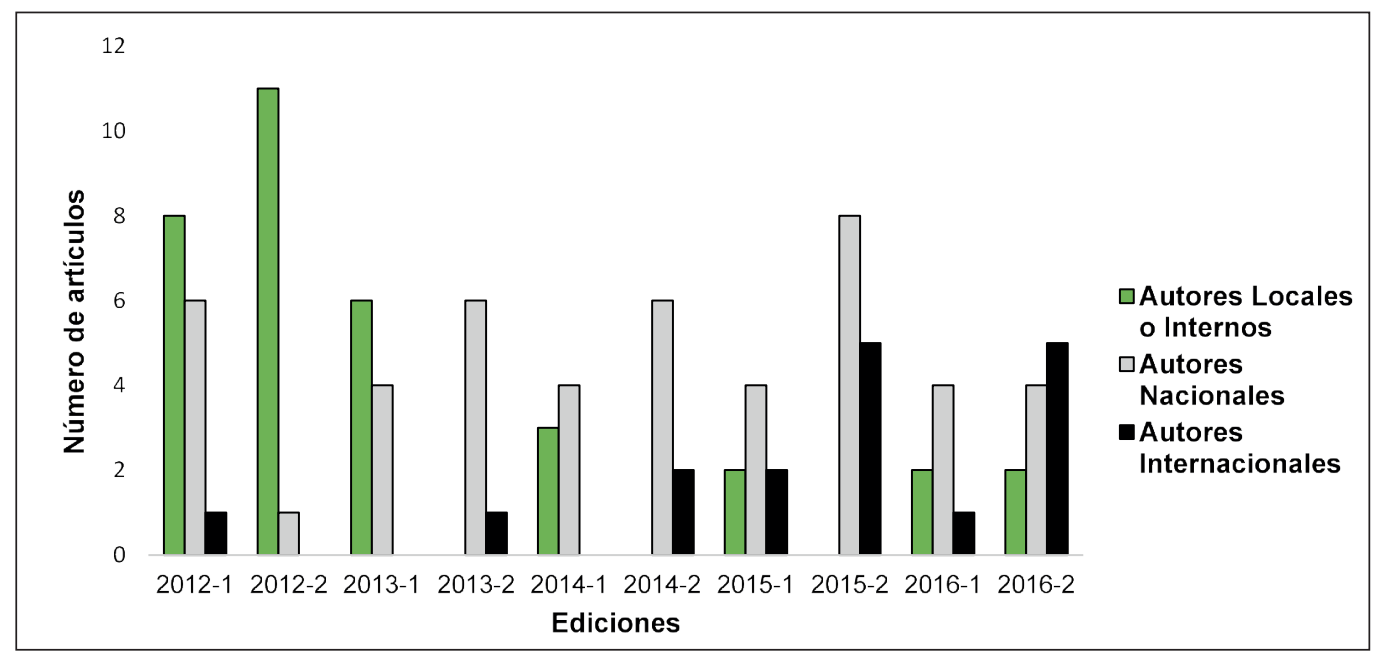

Figura 2. Número de artículos publicados en cada una de las ediciones, de acuerdo a la afiliación institucional del primer autor.

Las instituciones con dos o más colaboraciones, teniendo en cuenta la afiliación del primer autor, fueron: la Universidad del Magdalena con 51,56\%, la Universidad de Cartagena y la Universidad Cooperativa de Colombia con un 12,50\%, el Instituto de Investigación del Comportamiento Humano con 6,25\% y la Corporación Universitaria Rafael Núñez con un 4,69\%; las restantes coincidieron con un porcentaje de 3,13 (Tabla 1).

Tabla 1. Instituciones con dos o más artículos publicados en la revista Duazary, de acuerdo a la primera firma del mismo.

\begin{tabular}{|l|c|c|}
\hline \multicolumn{1}{|c|}{ Instituciones con dos o más publicaciones } & Número de artículos & Porcentaje \\
\hline Universidad del Magdalena & 33 & 51,56 \\
\hline Universidad de Cartagena & 8 & 12,50 \\
\hline Universidad Cooperativa de Colombia & 8 & 12,50 \\
\hline Instituto de Investigación del Comportamiento Humano & 4 & 6,25 \\
\hline Corporación Universitaria Rafael Núñez & 3 & 4,69 \\
\hline Universidad Simón Bolívar & 2 & 3,13 \\
\hline Universidad de Pamplona & 2 & 3,13 \\
\hline Universidad Metropolitana de Barranquilla & 2 & 3,13 \\
\hline Universidad Nacional de Colombia & 2 & 3,13 \\
\hline Total & 64 & 100 \\
\hline
\end{tabular}


El índice de colaboración (cociente entre el número de firmas y el número de trabajos) de cada una de la ediciones osciló entre un mínimo de 2,75 y un máximo de 4,18 autores por artículos, para las ediciones 2014-2 y 2016-2 respectivamente (Figura 3), siendo la media de estas de 3,51. Se destaca que sólo un artículo presentó 10 autores.

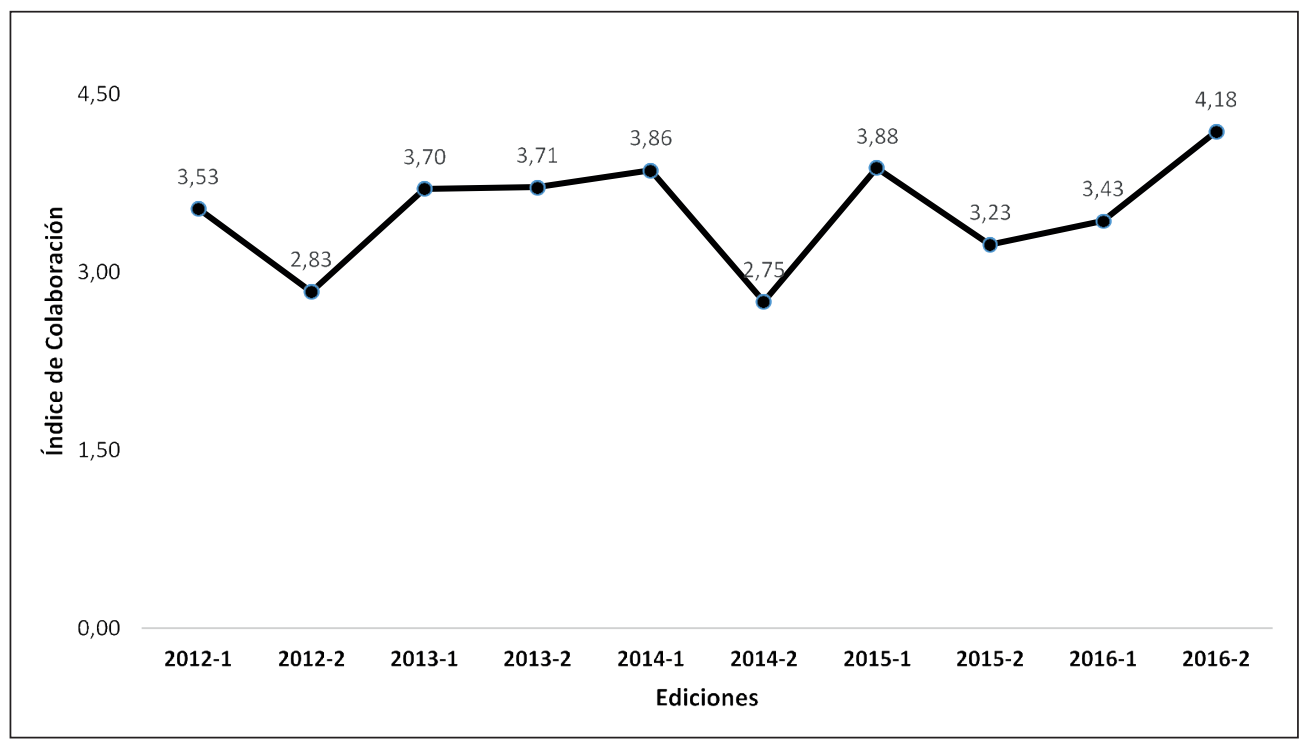

Figura 3. Índice de colaboración de cada una de las ediciones en estudio.

En lo que respecta a la distribución por disciplinas, se encontró que la más frecuente fue ciencias sociobiomédicas con $22(22,45 \%)$ publicaciones, seguida de odontología, 16 (16,33\%); enfermería, 11 (11,22\%); otros temas de medicina clínica, seis (6,12\%), psiquiatría, cinco $(5,10 \%)$; entre otros. Hay que señalar que estas disciplinas se clasifican en las áreas de medicina clínica y ciencias de la salud. Estos resultados se presentan en la siguiente tabla (Tabla 2).

Tabla 2. Temática tratada en los cada uno de los artículos publicados en la revista Duazary, durante los cinco años del estudio.

\begin{tabular}{|c|c|c|c|}
\hline Área & Disciplina & $\begin{array}{c}\text { Número de } \\
\text { artículos }\end{array}$ & Porcentaje \\
\hline \multirow{12}{*}{ Medicina clínica } & Oftalmología & 2 & 2,04 \\
\hline & Respiratoria & 2 & 2,04 \\
\hline & Odontología & 16 & 16,33 \\
\hline & Neurología Clínica & 3 & 3,06 \\
\hline & Otros temas de medicina clínica & 6 & 6,12 \\
\hline & Psiquiatría & 5 & 5,10 \\
\hline & Ortopedia & 2 & 2,04 \\
\hline & Medicina general e interna & 4 & 4,08 \\
\hline & Oncología & 1 & 1,02 \\
\hline & Pediatría & 1 & 1,02 \\
\hline & Endocrinología y metabolismo & 2 & 2,04 \\
\hline & Urología y nefrología & 2 & 2,04 \\
\hline
\end{tabular}


Continuación Tabla 2.

\begin{tabular}{|l|l|c|c|}
\hline \multirow{2}{*}{ Área } & \multicolumn{1}{|c|}{ Disciplina } & $\begin{array}{c}\text { Número de } \\
\text { artículos }\end{array}$ & Porcentaje \\
\hline \multirow{4}{*}{ Medicina clínica } & Medicina química & 1 & 1,02 \\
\cline { 2 - 4 } & Hematología & 2 & 2,04 \\
\cline { 2 - 4 } & Geriatría & 1 & 1,02 \\
\hline \multirow{5}{*}{ Ciencias de la salud } & Salud ocupacional & 2 & 2,04 \\
\cline { 2 - 4 } & Ciencias sociobiomédicas & 22 & 22,45 \\
\cline { 2 - 4 } & Enfermería & 11 & 11,22 \\
\cline { 2 - 4 } & Nutrición y dietas & 1 & 1,02 \\
\cline { 2 - 4 } & Epidemiología & 2 & 2,04 \\
\cline { 2 - 4 } & Salud pública & 5 & 5,10 \\
\cline { 2 - 4 } & Políticas de salud y servicios & 1 & 1,02 \\
\cline { 2 - 4 } & Enfermedades infecciosas & 1 & 1,02 \\
\hline Otras ciencias médicas & Otras ciencias médicas & 1 & 1,02 \\
\hline Biotecnología en salud & Biotecnología relacionada con la salud & 1 & 1,02 \\
\hline Medicina básica & Patología & 98 & 100 \\
\hline Total & & 1 & 2 \\
\hline
\end{tabular}

Con el propósito de realizar una revisión de las citaciones recibidas por los artículos publicados en la revistas, se utilizó el programa informático Publish Or Perish, creado por la profesora Anne-Wil Harzing y disponible de manera gratuita en el portal que lleva por nombre su apellido. De esta manera, se encontró que la revista recibió, en este último quinquenio, 85 citas, para un promedio de 0,9 por artículo. Se destacan 11 artículos, los cuales han recibido tres o más citas, con un valor máximo de seis (Tabla 3).

Tabla 3. Artículos más citados entre los años 2012-2016 en la revista Duazary.

\begin{tabular}{|c|c|c|}
\hline Título & Año de publicación & Número de citas \\
\hline $\begin{array}{l}\text { Calidad del dormir, insomnio y rendimiento académico en } \\
\text { estudiantes de Medicina }{ }^{14}\end{array}$ & 2014 & 6 \\
\hline $\begin{array}{l}\text { Estilos de vida y factores sociodemográficos asociados en } \\
\text { adolescentes escolarizados del distrito de Santa Marta, Colombia }{ }^{15}\end{array}$ & 2014 & 6 \\
\hline El psicólogo educativo y su quehacer en la institución educativa ${ }^{16}$ & 2013 & 6 \\
\hline $\begin{array}{l}\text { Ideación suicida, depresión y autoestima en adolescentes escolares } \\
\text { de Santa Marta }{ }^{17}\end{array}$ & 2015 & 4 \\
\hline $\begin{array}{l}\text { Diferencias de las prácticas sexuales entre hombres y mujeres } \\
\text { universitarios }{ }^{18}\end{array}$ & 2014 & 4 \\
\hline Bullying: un fenómeno por transformar ${ }^{19}$ & 2012 & 4 \\
\hline $\begin{array}{l}\text { Factores relacionados con la depresión en pacientes pediátricos con } \\
\text { cáncer y participación de la enfermera en su detección }{ }^{20}\end{array}$ & 2015 & 3 \\
\hline La discapacidad y su estado actual en la legislación colombiana ${ }^{21}$ & 2015 & 3 \\
\hline
\end{tabular}


Continuación Tabla 3.

\begin{tabular}{|l|c|c|}
\hline \multicolumn{1}{|c|}{ Título } & Año de publicación & Número de citas \\
\hline $\begin{array}{l}\text { Conocimientos y prácticas sobre enfermedades diarreicas y } \\
\text { respiratorias entre madres de una institución de salud }{ }^{22}\end{array}$ & 2014 & 3 \\
\hline $\begin{array}{l}\text { Factores asociados al ejercicio de la sexualidad de las y los } \\
\text { adolescentes escolarizados de Cartagena-Colombia 2008 }\end{array}$ & 2013 & 3 \\
\hline $\begin{array}{l}\text { Características de inteligencia emocional y género en estudiantes } \\
\text { de Psicología y Administración de Empresas de una universidad } \\
\text { pública de Santa Marta, Colombia: un estudio piloto }\end{array}$ & 2012 & 3 \\
\hline $\begin{array}{l}\text { Prevalencia del suicidio femenino en el departamento del Magdalena } \\
\text { (Colombia): } 2004-2010^{25}\end{array}$ & 2012 & 3 \\
\hline
\end{tabular}

Fuente: Publish or Perish

Por último, para el caso del idioma de publicación de los artículos, se encuentra que la revista publica por lo general, en español. Solo se publicaron dos artículos en inglés durante el quinquenio, lo que equivale al 1,16\%.

\section{DISCUSIÓN}

La Revista Duazary es una publicación que en los últimos años ha tenido un crecimiento considerable, que se denota al revisar los datos publicados en esta investigación. Es por ello, que se hace necesario aclarar que los resultados obtenidos solo representan validez para Duazary. No obstante, pueden tomarse como insumo para otros estudios de producción científica colombiana y latinoamericana, aunque considerando el periodo analizado. La revisión de indicadores como: idioma, afiliación institucional, tipología, disciplina tratada, etc. permitió establecer que los artículos publicados están en concordancia con lo expuesto en su enfoque y alcance:

Duazary es una revista arbitrada por pares nacionales e internacionales, que tiene como propósito difundir investigaciones originales e inéditas relacionadas con temas salud pública, cirugía, especialidades clínicas, epidemiologia, educación médica y demás ramas afines que impactan. Publica artículos desde perspectivas amplias e integrales hasta manuscritos que presenten aspectos específicos y concretos de áreas clínicas que contribuyen con la comprensión del proceso saludenfermedad en el ámbito local, nacional e internacional. Su prioridad temática se enfoca en temas de salud de interés para el Caribe y Latinoamérica.
En lo relacionado con el idioma, se coincidió con lo expuesto por Castiel y Sanz-Valero ${ }^{26}$, y Sanz-Valero et $\mathrm{a}^{27}$, quienes resaltan en sus investigaciones que el español y, en menos proporción, el portugués son los idiomas más utilizados a la hora de escribir publicaciones científicas en América Latina, asegurando que es una constante en las revistas de ciencias de la salud de la región. Esto puede tener como explicación el hecho de que aquellos autores que tienen la posibilidad de redactar sus artículos en inglés, ya sea por iniciativa propia o por apoyo de la institución a la que se encuentran adscritos, tiendan a remitir sus escritos a revistas de alto impacto, que por lo general son anglófonas; muy a pesar de que esto suponga un costo adicional a la hora de consultar sus manuscritos una vez hayan sido publicados ${ }^{3}$. Por lo anterior, para solventar la baja tasa de recepción de artículos en inglés, algunas revistas han optado por adquirir un servicio de traducción a este idioma; dicho de otra forma, se publican los manuscritos en simultáneo, tanto en español como en inglés, por un tiempo determinado, con el propósito de que a mediano plazo puedan recibir en mayor proporción artículos escritos en este segundo idioma o convertirse exclusivamente al inglés ${ }^{28}$. Esta estrategia está siendo contemplada por el equipo editorial de la revista Duazary, con la intención de captar más publicaciones escritas en este idioma y poder postularse a índices y bases bibliográficas que exigen este tipo de contribuciones.

Respecto a la tipología de los manuscritos, más del 50\% del total de los registrados para este estudio corresponden a artículos originales 0 , como los denomina Colciencias, a artículos de investigación científica y tecnológica, lo que 
coincide con el trabajo de Restrepo-Valencia et al. ${ }^{29}$ que obtuvo un valor cercano al $45 \%$, lo que se supone es un buen indicador, ya que este tipo de documentos, junto a los de revisión, son los más consultados y citados en una publicación. Sin embargo, esto contrasta con lo hallado por Monteserín et al ${ }^{28}$, en donde el $49 \%$ de la producción autoral proviene de casos clínicos, lo que según este autor puede estar supeditado al hecho de que la mayoría de los autores están vinculados a centros hospitalarios, lo cual es el resultado de su labor principal.

La distribución temporal de los artículos publicados mostró que el número de estos ha disminuido gradualmente en relación con los años 2012 y 2013, aunque el elevado número de publicaciones en estos dos años se debía a que la mayoría de los artículos eran de origen local. Esto cambió en el 2014, a partir de allí las contribuciones internas fueron menores a las nacionales e internacionales, inclusive en la edición 2015-2 no tuvieron representantes, lo cual va en concordancia con lo exigido por Redalyc ${ }^{30}$ en su criterio de «Exogeneidad en publicación», donde expresa que al menos el $70 \%$ de las contribuciones deben ser externas a la institución que edita la revista.

El índice de colaboración medio fue de 3,51, lo cual está dentro del rango de la literatura latinoamericana, que se ubica entre 3 y 3,5 , aunque muy por debajo de revistas del top 10 de salud a nivel global ${ }^{31}$. Este índice se incrementó notablemente en las tres últimas ediciones de la revista, lo que es un buen indicador pues refleja la consolidación de las redes de trabajo interinstitucionales y, además, es evidencia de profesionalización de la comunidad científica. Otra explicación puede ser la constante evolución de la ciencia, que hace que las investigaciones sean más complejas, por lo que se requiere de investigadores asesores y de la relación con otros equipos de investigación interinstitucionales ${ }^{32}$.

El análisis de las instituciones reveló que un tercio de las publicaciones de la revista, en estos cinco años, proviene de la Universidad del Magdalena (Entidad editora), lo cual es un valor que refleja la escasa recepción de artículos externos que tenía Duazary en los dos primeros años de este quinquenio, donde más del $80 \%$ de los escritos con afiliación interna fueron publicados en ese lapso. Esta situación se ha ido subsanando gradualmente con el fuerte compromiso que ha tenido su equipo editorial en incrementar la visibilidad a nivel nacional e internacional, a través de la aplicación a un número considerable de bases bibliográficas, además de otra serie de estrategias editoriales. Otro punto a resaltar es que casi todas las instituciones firmantes son universidades, lo que está acorde con su responsabilidad de formar investigadores capaces de generar nuevos conocimientos que contribuyan al desarrollo del país y de la región ${ }^{33}$.

Para finalizar, por áreas temáticas se destaca ciencias de la salud y medicina clínica, con más del $95 \%$ de los trabajos publicados, lo que coincide con otros estudios de Latinoamérica ${ }^{33,34}$, en donde predominaron este tipo de temáticas que ponen a las ciencias médicas como una de las más productivas a nivel mundial.

\section{REFERENCIAS BIBLIOGRÁFICAS}

1. Camps D. Limitaciones de los indicadores bibliométricos en la evaluación de la actividad científica biomédica. Colomb. Med. 2008 Mar; 39(1):74-9.

2. López-Piñero JM, Terrada ML. Los indicadores bibliométricos y la evaluación de la actividad médico científica (I). Usos y abusos de la bibliometría. Med Clin (Barc).1992; 98(2):64-8.

3. Tomás-Casterá V, Sanz-Valero J, Wanden-Berghe C. Estudio bibliométrico de la producción científica de la Revista de Nutrição a través de la Red SciELO (2001 a 2007). Rev. Nutr. 2010 Oct; 23(5):791-9.

4. Patrón C, López Jordi M, Piovesan S, Demaría B. Análisis bibliométrico de la producción científica de la revista Odontoestomatología. Odontoestomatología. 2014 May;16(23): 34-43.

5. Camps D, Samar ME, Ávila RE, Recuero Y. Estudio bibliométrico de un volumen de la revista Archivos de Medicina. Arch Med. 2006; 2(3).

6. Camps D, Recuero Y, Samar ME, Ávila R. Análisis bibliométrico de tesis de doctorado del área de las ciencias de la salud: Primera parte, Odontología. Rev Fac Cienc Med Univ Nac Córdoba. 2005; 62: 53-6.

7. Bordons M, Gómez CI. La actividad científica española a través de indicadores bibliométricos en el período 1990-93. Rev Gen Inf Doc. 1997; 7: 69-86.

8. Rousseau R. Indicadores bibliométricos y econométricos en la evaluación de instituciones científicas. ACIMED. 2001; 9:23-9.

9. Amsterdamska O, Leydesdorff L. Citations: indicators of significance? Scientometrics. 1989; 15 (5-6): 444-71.

10. López AA, Núñez C, Vicente-Herrero MT, Monroy N, Sarasibar H, Tejedo E. Análisis bibliométrico de la productividad científica de los artículos originales relacionados con salud laboral publicados por diferentes revistas españolas entre los años 1997 y 2006. Medicina Balear. 2008; 23(1):17-23.

11. Tomás-Casterá V, Sanz-Valero J, Wanden-Berghe C. Estudio bibliométrico de la producción científica y uso de la revista chilena de Nutrición a través de la red Scielo (2002 a 2007). Rev. chil. nutr. 2010 Sep; 37(3):330-9. 
12. Rojas-Sola JI, San-Antonio-Gomez C. Análisis bibliométrico de las publicaciones científicas mexicanas en la categoría Engineering Chemical de la base de datos Web of Science (1997-2008). Revista Mexicana de Ingeniería Química. 2010; 9(3):231-40.

13. Organización para la Cooperación y el Desarrollo Económicos (OCDE). "Propuesta de Norma Práctica para encuestas de Investigación y Desarrollo Experimental. Manual de Frascati”; 2002. Disponible en: http:// www.idi.mineco.gob.es/stfls/MICINN/Investigacion/ FICHEROS/ManuaFrascati-2002_sp.pdf

14. Monterrosa A, Ulloque L, Carriazo S. Calidad del dormir, insomnio y rendimiento académico en estudiantes de Medicina. Duazary. 2014 Dic;11(2):85-97.

15. Ferrel F, Ortiz A, Forero L, Herrera M, Peña Y. Estilos de vida y factores sociodemográficos asociados en adolescentes escolarizados del Distrito de Santa Marta, Colombia. Duazary. 2014 Dic;11(2):105-14.

16. Arvilla-Ropain AR, Palacio-Orozco LP, Arango-Gonzáles CP. El psicólogo educativo y su quehacer en la institución educativa. Duazary. 2011; 8(2):258-61.

17. Ceballos-Ospino G, Suarez-Colorado Y, SuescúnArregocés J, Gamarra-Vega L, Gonzalez K, SoteloManjarres A. Ideación suicida, depresión y autoestima en Adolescentes escolares de Santa Marta. Duazary. 2015 Jun;12(1):15-22.

18. Martínez J, Maya N, Parada L, Duarte K. Diferencias de las prácticas sexuales entre hombres y mujeres, en estudiantes universitarios durante el primer semestre de 2013. Duazary. 2013 Dic;10(2):112-8.

19. Rodríguez-Díaz A, Mejía-Moreu Y. Bullying: un fenómeno por transformar. Duazary. 2012; 9(1):98-104.

20. Velásquez-Silva S, Zuluaga-Sarmiento L. Factores relacionados con la depresión en pacientes pediátricos con cáncer y participación de la enfermera en su detección. Duazary. 2015 Dic; 12 (2): 164-73.

21. Martínez-Rozo A, Uribe-Rodríguez A, VelázquezGonzález H. La discapacidad y su estado actual en la legislación colombiana. Duazary. 2015 Jun; 12(1):49-58.

22. Lapeira P, Salazar D. Conocimientos y prácticas sobre enfermedades diarreicas y respiratorias entre madres de una institución de salud. Duazary. 2014 Jun; 11(1):36-40.

23. Díaz CE, Rodríguez W, Lara L, Matute R, Ariza H. Factores asociados al ejercicio de la sexualidad de las y los adolescentes escolarizados de Cartagena - Colombia 2008. Duazary. 2014 Dic; 10(2):88-97.

24. Suárez-Colorado Y, Guzmán-García K, Medina-Alfonso L, Ceballos-Ospino G. Características de inteligencia emocional y género en estudiantes de Psicología y Administración de Empresas de una universidad pública de Santa Marta, Colombia: un estudio piloto. Duazary. 2012; $9(2): 132-9$.

25. Pérez-Manjarrez D, Calderón-Araujo A. Prevalencia del suicidio femenino en el departamento del Magdalena (Colombia): 2004-2010. Duazary. 2012; 9(1):87-93.

26. Castiel L, Sanz-Valero J. Entre fetichismo e sobrevivência: 0 artigo cientifico é uma mercadoria acadêmica? Cad Saude Publica. 2007; 23(12):3041-50.

27. Sanz-Valero J, Casterá Vicente T, Wanden-Berghe C. Estudio bibliométrico de la producción científica publicada por la Revista Panamericana de Salud Pública/ Pan American Journal of Public Health en el período de 1997 a 2012. Rev Panam Salud Pública. 2014 Feb; 35(2): 81-8.

28. Monteserín B, Junquera LM, Cordón JA, Llorente S. Análisis bibliométrico de la producción científica de la Revista Española de Cirugía Oral y Maxilofacial durante el periodo 2005-2011. Rev Esp Cir Oral Maxilofac. 2014; 36(4):156-63.

29. Restrepo-Valencia L, Cano A, Castañeda C, Sánchez R, González-Ariza SE. Análisis de la producción científica de la revista CES Odontologia en los últimos 10 años. Rev. CES Odont 2015; 28(2): 119-31.

30. Redalyc. Metodología de evaluación de revistas Redalyc. Disponible en: http://www.redalyc.org/redalyc/media/ redalyc_n/estaticasredalyc/Criterios/criterios.html

31. Coimbra CE. Produção científica em saúde pública e as bases bibliográficas internacionais. Cad Saude Pública. 1999; 15:883-8.

32. Camps D. Estudio bibliométrico general de colaboración y consumo de la información en artículos originales de la revista Universitas Médica, periodo 2002 a 2006. Universitas Med 2007; 48(4):358-65

33. Diaz-Mujica D. Análisis bibliométrico de la revista Anales Venezolanos de Nutrición. An Venez Nutr. 2010 Jun; 23(1):34-41.

34. Alvis-Guzmán N, De La Hoz-Restrepo F. Producción científica en ciencias de la salud en Colombia, 19932003. Rev Salud Pública. 2006; 8(1):25-37. 